\section{ADDTREE/P: A PASCAL program for fitting additive trees based on Sattath and Tversky's ADDTREE algorithm}

\author{
JAMES E. CORTER \\ Stanford University, Stanford, California 94305
}

Sattath and Tversky (1977) discuss the use of additive trees to represent proximity data and introduce a program, ADDTREE, for fitting such tree models to data. The program is based on an efficient and robust algorithm, but the code is written in CDC FORTRAN and has run into portability problems. Since efficiency apparently was emphasized at the expense of transparency of design, debugging is difficult. The present program, ADDTREE/P, is an attempt to incorporate the basic algorithm develop by Sattath and Tversky into a program written in the PASCAL language, emphasizing clarity of design, modifiability, and portability. New analysis and output options and statistics have been added to make the program more useful.

Input. The basic input for the program is a matrix of proximity data, that is, data interpretable as distances or similarities between a set of objects (such as similarity ratings, confusion data, or correlations). The actual input file should contain: an analysis and output options control line, a comment line, a data description line, the actual data in matrix form, and an optional list of object names. The data matrix may be either similarities or dissimilarities, and may be either in full or lower half matrix form.

Output. The basic output is a graph of the fitted tree structure, along with parameter estimates and some statistics measuring fit of the model. More specifically, the program prints: a description of any transformations applied to the data, a list of each node created along with the length of the arc connecting it with its parent and the number of any children nodes, the actual tree graph (in rooted form), and the value of several fit statistics (stress1, stress2, squared monotonic and linear correlations). Many output options are allowed for: printing of the raw data, the transformed data, obtained model distances, and residual distances, specification of the order of the leaves of the tree, numbering and labeling of the leaves, and so on.

Overview of Algorithm. The matrix of proximities is input and converted to distance-like numbers. This involves symmetrizing the matrix by averaging the two halves, converting similarities into dissimilarities by multiplication by -1 , and addition of a constant to satisfy the triangle inequality. The data then satisfy the axioms of a metric space.

Each quadruple of objects is then examined, and the tree inequality (also known as the additive inequality, or the 4-point condition) is checked. When the six distances among the four objects are sorted into the three sums of the tree inequality, the pairs of objects in the smallest of the sums receive a "neighbor score" of 2 , those pairs in the next smallest sum receive a score of 1 , and those in the largest receive a 0 . This procedure, repeated over all possible quadruples of objects, gives a robust measure of closeness between each pair of objects (the neighbor score for that pair, summed over all quadruples of objects).

Using the matrix of neighbor scores, the nearest neighbor of each object is found. At least one pair of objects (corresponding to the largest neighbor score) must consist of mutual nearest neighbors; that is, the nearest neighbor of $i$ is $j$, and the nearest neighbor of $j$ is $i$. Those pairs of objects that are mutual nearest neighbors are marked to be joined or clustered together at this stage of the algorithm. In this way, up to half of the objects may be joined at each iteration of the tree construction.

Pairs of objects that are joined at one stage are considered a single object at the next iteration, and distances to all other objects are redefined as the weighted average of distances from the joined objects. The reduced matrix is then resubmitted to the checking of quadruples and the computation of neighbor scores. The entire process is repeated until only two or three objects remain.

Since estimation is done in parallel with the tree construction above, the only problem that remains is the selection of a root for the tree. An additive tree is best thought of as an unrooted or "free" tree. However, for plotting purposes, a root is chosen and the tree is displayed in rooted form. The root chosen is that point in the tree that maximizes a function of the lengths of its "children": in a sense, the "deepest" rooting.

The rooted tree constructed by this algorithm is a binary tree; that is, each node has one "parent" (with the exception of the root) and two "children." This results in the least squares estimates of the branches being easily computable as a simple function of the average distances between subtrees. If Objects $i$ and $j$ are neighbors in the tree, the estimate of the length of the arc connecting $i$ with the node defined by the joining of $i$ and $j$, defined as $1(i)$, is simply $1(i)=1 / 2[d(i, j)+d(i, K)$ - $d(j, K)]$, where $d(i, j)$ is defined as the (data) distance between $i$ and $j$, and $d(i, K)$ as the average distance between $i$ and all other objects in the tree $(\neq i, j)$. Estimates for interior arcs are based on a straightforward generalization of this formula, in which distances from Object $i$ are replaced by average distances from a subtree. Negative estimates, since they are inconsistent with the model, are set to zero.

Language. ADDTREE/P is written in standard PASCAL (Jensen \& Wirth, 1974) and has been tested on the following systems: a DEC TOPS-20 system, a 
VAX running UNIX, and an IBM 3033/370 with the PASCAL/VS compiler. The source code available by request will run without significant modification on all these systems.

Availability. A magnetic tape containing the source listings of the program and more complete documentation may be obtained by writing to James Corter or Amos Tversky at the Department of Psychology, Building 420, Stanford University, Stanford, California 94305.
A fee of $\$ 25$ is required to cover costs of the tape and postage.

\section{REFERENCES}

JENSEN, K., \& WIRTH, N. PASCAL user manual and report (2nd ed.). New York: Springer-Verlag, 1974.

Sattath, S., \& Tversky, A. Additive similarity trees. Psychometrika, 1977, 42, 319-345.

(Accepted for publication February 13, 1982.) 